\title{
Injúria renal aguda na Unidade de Terapia Intensiva em um hospital do interior
}

\section{amazônico}

\author{
Acute kidney injury in the Intensive Care Unit of a hospital in the interior of the amazon region
}

Lesión renal aguda en una Unidad de Cuidados Intensivos de un hospital del interior de la región amazónica

Recebido: 07/08/2021 | Revisado: 11/08/2021 | Aceito: 12/08/2021 | Publicado: 15/08/2021

\author{
Jamyle Balla da Silva \\ ORCID: https://orcid.org/0000-0003-4402-8681 \\ Universidade do Estado do Pará, Brasil \\ E-mail: jamyle.balla@hotmail.com \\ Cilene Aparecida de Souza Melo \\ ORCID: https://orcid.org/0000-0003-1170-6466 \\ Universidade do Estado do Pará, Brasil \\ E-mail: souza_cilene927@hotmail.com \\ Thiago Gonçalves Barros \\ ORCID: https://orcid.org/0000-0002-8822-541X \\ Clínica de Doenças Renais, Brasil \\ E-mail: thiagogbarros@ hotmail.com
}

\begin{abstract}
Resumo
Objetivo: Identificar e analisar a injúria renal aguda na unidade de terapia intensiva em um hospital do interior amazônico. Metodologia: Trata-se de um estudo observacional, descritivo, prospectivo, transversal com abordagem quantitativa de uma amostra de 30 indivíduos, realizada no período de maio a junho de 2021 em um hospital de referência no interior amazônico. Resultados: Observou-se que o sexo prevalente foi masculino (63\%), e que 57\% tinham mais de 55 anos. Prevaleceu o tempo de internação de até 7 dias (70\%). O principal diagnóstico de admissão foi TCE/politrauma. Ratificou-se a HAS e DM como principais fatores de risco e que mais de $50 \%$ da amostra apresentou alterações glicêmicas e pressóricas durante a internação. Notou-se que $70 \%$ dos pacientes precisaram de VM e 63,40\% de DVA. Os estágios 2 e 3 do KDIGO estiveram presentes em $70 \%$ da amostra. A principal medida terapêutica foi hidratação venosa e houve $40 \%$ de morte ao final deste estudo. Conclusão: Constatou-se que indivíduos que tinham alguma doença crônica de base e que tinham hábitos de vida não saudáveis obtiveram pior score na classificação KDIGO e apresentaram desfecho desfavorável. Portanto, compreender o perfil dos indivíduos atendidos no serviço pode favorecer melhor atendimento médico, com maior rapidez diagnóstica e permitir otimização terapêutica, afim de reduzir os impactos na morbimortalidade induzidos pela injúria renal aguda na unidade de terapia intensiva.
\end{abstract}

Palavras-chave: Unidade de terapia intensiva; Insuficiência renal; Doença aguda.

\begin{abstract}
Objective: To identify and analyze acute kidney injury in an intensive care unit of a hospital in the interior of the Amazon. Methodology: This is an observational, descriptive, prospective, cross-sectional study, with a quantitative approach of a sample of 30 individuals, carried out from Mai to June 2021 in a reference hospital outside the interior of the Amazon. Results: It was observed that the most prevalent sex was male $(63 \%)$ and that $57 \%$ were over 55 years old. The length of stay of up to 7 days (70\%) prevailed. The main admission diagnosis of TBI / polytrauma. It was confirmed that SAH and DM were the main risk factors and that more than $50 \%$ of the sample changed blood glucose and blood pressure during hospitalization. $70 \%$ of patients needed MV and $63.40 \%$ needed VAD. Stages 2 and 3 of KDIGO were present in $70 \%$ of the sample. The main therapeutic measure was intravenous hydration and there was $40 \%$ death at the end of this study. Conclusion: It was contacted that individuals who had some underlying chronic disease and who had unhealthy lifestyle habits had the worst score in the KDIGO classification and had an unfavorable outcome. Therefore, understanding the profile of individuals assisted in the service can favor better medical care, with faster diagnosis and allow therapeutic optimization, in order to reduce the impacts on morbidity and mortality induced by acute kidney injury in the intensive care unit.
\end{abstract}

Keywords: Intensive care unit; Renal failure; Acute disease. 


\begin{abstract}
Resumen
Objetívo: Identificar y analizar la lesión renal aguda en la unidad de cuidados intensivos de un hospital del interior de la Amazonía. Metodología: Se trata de un estudio observacional, descriptivo, prospectivo, transversal con abordaje cuantitativo de una muestra de 30 individuos, realizado de mayo a junio de 2021 en un hospital de referencia en el interior de la Amazonía. Resultados: Se observó que el sexo más prevalente fue el masculino (63\%) y el 57\% tenían más de 55 años. Prevaleció la estancia de hasta 7 días (70\%). El principal diagnóstico de ingreso fue TCE / politraumatismo. Se constató que la HSA y la DM eran los principales factores de riesgo y más del $50 \%$ de la muestra presentaba alteraciones de la glucemia y de la presión arterial durante el ingreso. El $70 \%$ de los pacientes necesitaron VM y el 63,40\% necesitaron DAV. Las etapas 2 y 3 de KDIGO estuvieron presentes en el $70 \%$ de la muestra. La principal medida terapéutica fue la hidratación intravenosa y hubo un $40 \%$ de muerte al final de este estudio. Conclusión: Se contactó que los individuos que tenían alguna enfermedad crónica subyacente y que tenían hábitos de vida poco saludables tenían la peor puntuación en la clasificación KDIGO y tenían un resultado desfavorable. Por tanto, conocer el perfil de las personas atendidas en el servicio puede favorecer una mejor atención médica, con un diagnóstico más rápido y permitir una optimización terapéutica, con el fin de reducir los impactos en la morbimortalidad inducida por la lesión renal aguda en la unidad de cuidados intensivos.
\end{abstract}

Palabras clave: Unidad de cuidados intensivos; Insuficiencia renal; Enfermedad aguda.

\title{
1. Introdução
}

A Insuficiência Renal Aguda (IRA) é descrita na literatura como a perda súbita da função renal, caracterizada pela redução abrupta da taxa de filtração glomerular e/ou do volume urinário, resultando na incapacidade dos rins em excretar produtos nitrogenados e manter a homeostase de líquidos e eletrólitos no corpo (Lopes, et al, 2018).

Atualmente, o termo mais adequado para definir essa síndrome é Injuria Renal Aguda (IRA), proposto com o intuito de enfatizar que o processo contínuo de lesão renal se inicia muito antes que a perda renal da função de excreção possa ser detectada por exames laboratoriais, além de reafirmar a dinâmica existente ao prognóstico e ao acréscimo na mortalidade em decorrência de aumentos na creatinina sérica (Lopes, et al, 2018; Luft, et al, 2016).

Sabe-se que, a IRA é uma complicação corriqueira no âmbito hospitalar e a sua incidência varia de acordo com a condição clínica do paciente, sendo mais notória na Unidade de Terapia Intensiva (UTI), onde observa-se esta evolução em cerca de 35\% dos pacientes, com um acréscimo na morbimortalidade de 10 a 15 vezes (Guedes, et al, 2017).

Ressalta-se ainda que a injúria renal aguda no âmbito intensivo apresenta múltiplas causas, dentre as quais destacamse a hipoperfusão, principalmente em decorrência de quadros sépticos e hipovolemia, a exacerbação de doenças crônicas, em especial hipertensão arterial sistêmica e diabetes mellitus, e ao uso prolongado de nefrotóxicos, como os anti-inflamatórios não esteroidais. Esses eventos culminam em lesões que causam o decréscimo célere da função dos néfrons, que, se não revertido rapidamente, pode tornar-se irreversível ou levar o paciente ao óbito (Souza, et al, 2017).

Nesse sentido, o tratamento da IRA depende da causa de base e do grau de acometimento da doença no paciente, de tal forma que as condutas iniciais devem permear a correção de volemia, a manutenção do equilíbrio eletrolítico, o controle das manifestações urêmicas, além de prover um rigoroso controle hidroeletrolítico e nutricional, bem como a correção do distúrbio acidobásico, caso existam. Isso é fundamental para evitar a alta letalidade dessa complicação, que varia entre 50 a $90 \%$ na unidade de terapia intensiva (Souza, et al, 2017; Guedes, et al, 2017).

Por conseguinte, quando as condutas terapêuticas supracitadas se tornam insuficientes ou incapazes de manterem uma condição clínica compatível com a vida, a terapia renal substitutiva deve ser implementada através da diálise peritoneal ou da hemodiálise. Ressalta-se que, a hemodiálise apresenta-se como principal forma de terapia substitutiva, apresentando duração variável entre 3 a 4 horas por sessão e, em geral, realizada 3 vezes por semana (Sesso, et al, 2016; Orlandi, et al, 2014; Cerqueira et al, 2014) e que tal realidade exige tecnologia avançada e um aumento progressivo de recurso terapêuticos (Hsiao \& Lima, 2015; Duarte, et al., 2016; Luft, et al, 2016).

Por fim, este trabalho objetiva traçar o perfil dos doentes acometidos por IRA, identificar os fatores de risco associados e seu desfecho em uma unidade de terapia intensiva em um hospital do interior amazônico. 


\section{Metodologia}

Trata-se de um estudo observacional, descritivo, prospectivo, transversal com abordagem quantitativa (Ludke e Andre, 2013). Foi realizado no município de Marabá no estado do Pará, no Hospital Regional do Sudeste do Pará - Marabá (PA), administrado pela empresa Pró Saúde. Ressalta-se que a pesquisa foi aprovada pelo Comitê de Ética em Pesquisa via Plataforma Brasil (CAAE: 38556520.1.0000.8607).

Antes de qualquer manipulação do participante, este ou seu responsável legal foi orientado sobre a pesquisa, seus riscos e benefícios e assinou o Termo de Consentimento Livre e Esclarecido.

Quanto a amostra, participaram desse estudo 30 indivíduos. Os critérios de inclusão foram: idade igual ou superior a 18 anos, internados na UTI por um período superior a 48 horas, no período de maio a junho de 2021, e que desenvolverem IRA no período de internação.

A coleta de dados se deu por meio de um questionário contendo perguntas objetivas, a saber: sexo, idade, tempo de internação na UTI, diagnóstico de entrada, presença de doenças crônicas prévias (Hipertensão arterial sistêmica e Diabetes Mellitus) e tabagismo. Além disso, foram coletados do prontuário do participante os seguintes tópicos: classificação da IRA segundo o KDIGO, nível glicêmico e pressórico, oxigenoterapia, uso de ventilação mecânica, uso de drogas vasoativas, uso de terapia substitutiva, bem como o desfecho clínico do paciente.

Após a realização da coleta das amostras, a elaboração do banco de dados e da análise estatística foi executada pelo programa Biostat 5.3. Por fim, foram realizadas análises descritivas, informando valores absolutos e percentuais de todos os dados analisados (Estrela, 2018).

\section{Resultados e Discussão}

Após a análise dos dados, observou- se que a maior proporção era do sexo masculino (63\%), em relação ao feminino (37\%). Isso corrobora com um estudo realizado por Dallacosta e Ferrari. (2018), em que 70\% da amostra com IRA era composta por homens. Isso se justifica em decorrência da menor frequência de cuidados alimentares e maior sedentarismo, consumo de álcool e tabagismo dos homens quando comparado as mulheres. Com relação a idade, a maior concentração esteve na faixa etária maior do que 55 anos (57\%) (Tabela 1). Este estudo está de acordo com os demais avaliados, visto que a idade avançada é reconhecida como um preditor independente para o desenvolvimento de IRA (Triquez e Dallacosta, 2012; Peres et al, 2015; Lopes et al, 2018).

Por conseguinte, 44\% dos indivíduos eram procedentes de Marabá. Infere-se tal realidade em decorrência da localização do hospital de referência onde este estudo foi realizado. Quanto ao tempo de internação, observou-se que 70\% estiveram internados por até 7 dias, seguidos por 8 a 15 dias (26,70\%) e apenas 3,30\% da amostra esteve internada por mais de 15 dias (Tabela 1). Os dados apresentados nesse estudo não são ratificados por outros autores, visto que, em geral, pacientes que desenvolveram injúria renal aguda tiveram maior tempo de internação, uma vez que, a cada dia adicional de internação, o risco de IRA aumenta cerca de 12,6\%. Presume-se com isto que o tempo de internação esteja associado as condições de gravidade, às comorbidades e à dependência ventilatória. (Prece et al, 2016; Benichel, \& Meneguim, 2018; Côrrea, 2020)

Quando analisados os diagnósticos de admissão (Tabela 1), observou-se que a principal causa foi politrauma/TCE, seguido de infarto agudo do miocárdio (IAM) e acidente vascular cerebral (AVC). Além disso, destacando-se as causas de IRA, verificou-se que as causas pré- renais foram as mais prevalentes $(73,40 \%)$. Dentre essas, a hipovolemia consiste em uma das principais causas de injúria renal aguda nos pacientes vítimas de TCE e politrauma, uma vez que, em geral, esses indivíduos perdem volume expressivo de sangue, resultando em uma redução direta da perfusão renal, ocasionando a redução da taxa de filtração glomerular e da eliminação das escórias renais, levando ao aumento de ureia e creatinina séricas, que culminam na IRA (Santos e Marinho, et al 2013; Rodriguez et al, 2016). Ademais, estudos demonstram que indivíduos com 
AVC e IAM que evoluem com IRA geralmente apresentam complicações advindas do diabetes e da hipertensão arterial sistêmica, como a nefropatia diabética, insuficiência cardíaca e lesões da microcirculação que somam expressivamente no maior risco de IRA durante a internação hospitalar desses pacientes (Chao-Yuan et al, 2020).

Tabela 1: Pacientes com injuria renal aguda, variáveis epidemiológicas, Marabá, 2021.

\begin{tabular}{|c|c|c|}
\hline Faixa etária & $\mathbf{F a}$ & Fr \% \\
\hline $18-40$ & 9 & $30 \%$ \\
\hline $41-55$ & 4 & $0,13 \%$ \\
\hline$>55$ anos & 17 & $57 \%$ \\
\hline Sexo & $\mathbf{F a}$ & Fr \% \\
\hline Masculino & 19 & $63 \%$ \\
\hline Feminino & 11 & $37 \%$ \\
\hline Procedência & Fa & Fr \% \\
\hline Bom Jesus & 2 & $6,60 \%$ \\
\hline Canãa dos Carajás & 1 & $3,30 \%$ \\
\hline Curionópolis & 2 & $6,60 \%$ \\
\hline Eldorado de Carajás & 2 & $6,60 \%$ \\
\hline Goianésia & 1 & $3,30 \%$ \\
\hline Itupiranga & 1 & $3,30 \%$ \\
\hline Marabá & 13 & $44 \%$ \\
\hline Novo Repartimento & 4 & $13,50 \%$ \\
\hline Parauapebas & 1 & $3,30 \%$ \\
\hline Rondon & 1 & $3,30 \%$ \\
\hline Santo Antônio & 1 & $3,30 \%$ \\
\hline Tucuruí & 1 & $3,30 \%$ \\
\hline Tempo de Internação & Fa & Fr \% \\
\hline 1 a 7 dias & 21 & $70 \%$ \\
\hline 8 a 15 dias & 8 & $26,70 \%$ \\
\hline$>$ de 15 & 1 & $3,30 \%$ \\
\hline Diagnóstico de admissão & $\mathbf{F a}$ & Fr \% \\
\hline Acidente vascular cerebral (AVC) & 5 & $16,75 \%$ \\
\hline Pneumonia & 3 & $10 \%$ \\
\hline Infarto agudo do miocárdio (IAM) & 5 & $16,75 \%$ \\
\hline Hemorragia digestiva & 4 & $13,40 \%$ \\
\hline Choque séptico & 1 & $3,30 \%$ \\
\hline TCE/politrauma & 6 & $20 \%$ \\
\hline Piodermite séptica & 1 & $3,30 \%$ \\
\hline Pielonefrite & 1 & $3,30 \%$ \\
\hline Urolitíase & 2 & $6,60 \%$ \\
\hline Doenças da tireóide & 1 & $3,30 \%$ \\
\hline Leshimaniose viceral & 1 & $3,30 \%$ \\
\hline Causa da IRA & $\mathbf{F a}$ & Fr \% \\
\hline Pré-Renal & 22 & $73,40 \%$ \\
\hline Renal & 6 & $20 \%$ \\
\hline Pós-Renal & 2 & $6,60 \%$ \\
\hline
\end{tabular}

Fonte: Acervo da pesquisa.

Além disso, quando analisados os dados referentes a presença de fatores de risco associados a injúria renal aguda (Tabela 2), verificou-se que 63,40\% da amostra apresentava hipertensão arterial sistêmica como comorbidade prévia a internação, que $46,60 \%$ eram diabéticos e que $66,60 \%$ eram tabagistas. Os fatores de risco observados na presente pesquisa corroboram com os demais estudos que abordam essa temática, visto que a HAS e a DM são as patologias de base mais comumente encontrada em indivíduos que evoluem com injúria renal aguda no ambiente de UTI (Bandiera, 2015). Associado a isso, o tabagismo promove alteração da microcirculação, levando a hipóxia celular, liberação de agentes oxidantes que resulta em um potencial agravante ao risco de dano renal (Guedes et al, 2017; Bandiera, 2015). 
Tabela 2: Pacientes com injúria renal aguda e comorbidades, Marabá, 2021.

\begin{tabular}{ccc}
\hline Hipertensão Arterial Sistêmica & Fa & Fr \% \\
\hline Sim & 19 & $63,40 \%$ \\
Não & 11 & $36,60 \%$ \\
\hline Diabetes Mellitus & Fa & Fr \% \\
\hline Sim & 14 & $46,60 \%$ \\
Não & 16 & $53,40 \%$ \\
\hline Tabagismo & Fa & Fr \% \\
\hline Sim & 20 & $66,60 \%$ \\
Não & 10 & $33,40 \%$ \\
\hline
\end{tabular}

Fonte: Acervo da pesquisa.

Com relação ao acompanhamento dos pacientes e ao suporte intensivo que foi necessário (Tabela 3), observou-se que mais da metade da amostra apresentou alteração glicêmica e pressórica, que $70 \%$ necessitou de ventilação invasiva e que 63,40\% precisou de droga vasoativa para manter estabilidade hemodinâmica. Os achados dessa pesquisa corroboram com a literatura de hiperglicemia e hipertensão arterial sistêmica como fatores de risco para doenças renais e sua respectiva morbimortalidade. De tal forma que, o controle e monitoramento dos níveis glicêmicos e pressóricos são estratégias fundamentais para prevenir ou reduzir a progressão de doenças renais (Kane-Gill et al, 2014; Gaião et al, 2016; Cardoso et al, 2017; Chao-Yuan et al, 2020).

Quanto ao uso de ventilação mecânica, sabe-se que seu uso gera consequências imediatas sobre a função renal, como a redução de 20 a $40 \%$ do fluxo urinário, recorrência de balanço hídrico positivo, retenção de sódio, queda do débito cardíaco e nas alterações humorais, que afetam direta ou indiretamente a função renal. Dessa forma, a ventilação mecânica é um importante preditor de mortalidade de pacientes com IRA na UTI. Essa realidade pode ser comprovada neste estudo, visto que grande parte dos pacientes que evoluíram com IRA utilizavam-se deste suporte ventilatório (Lopes et al, 2018).

Ademais, estudos demonstram que a utilização de droga vasoativa também é considerada fator de risco para o desenvolvimento de lesão renal aguda em pacientes críticos, fato também corroborado por esta pesquisa. Isso porque em função do mecanismo de vasoconstrição, essas drogas constituem uma das possíveis causas de injúria renal, ao predispor à lesão renal aguda, e, empregados por períodos prolongados associados com outros medicamentos nefrotóxicos, representam fator preditivo para nefrotoxicidade (Benichel \& Meneguim, 2018; Lopes et al, 2018). 
Tabela 3: Acompanhamento e suporte intensivo dos pacientes com injúria renal aguda, Marabá,2021.

\begin{tabular}{ccc}
\hline Nível glicêmico & Fa & Fr \% \\
\hline Alterado $(>180 \mathrm{mg} / \mathrm{dl})$ & 17 & $56,60 \%$ \\
Controlado & 13 & $43,40 \%$ \\
\hline Nível pressórico & Fa & Fr \% \\
\hline Alterado (>139x89mmhg) & 17 & $56,60 \%$ \\
Controlado & 13 & $43,40 \%$ \\
\hline Oxigenioterapia & Fa & Fr \% \\
\hline Sim & 2 & $6,60 \%$ \\
Não & 28 & $93,40 \%$ \\
\hline Ventilação mecânica & Fa & Fr \% \\
\hline Sim & 21 & $70 \%$ \\
Não & 9 & $30 \%$ \\
\hline Droga vasoativa & Fa & Fr \% \\
\hline Sim & 19 & $63,40 \%$ \\
Não & 11 & $36,60 \%$ \\
\hline
\end{tabular}

Fonte: Acervo da pesquisa.

Por conseguinte, após a classificação dos pacientes segundo os critérios diagnósticos para IRA - KDIGO (Kidney Disease Improving Global Outcomes) -, verificou-se que 36,60\% da amostra estava no Estágio 2, seguido por 33,40\% no Estágio 3 e apenas 30\% no Estágio 1 (Tabela 4). Isso corrobora com o estudo de Guedes et al (2017), que afirma que a evolução para injúria renal dos pacientes na UTI geralmente ocorre de forma rápida, sendo, por isso, notadamente diagnosticada em um estágio mais avançado.

Já com relação ao tratamento proposto, $73,40 \%$ foram submetidos a um incremento na hidratação venosa e $23,30 \%$ necessitaram de terapia de substituição renal, por meio da diálise (Tabela 4). Sabe-se que a conduta médica frente ao quadro de injuria renal aguda depende de inúmeros fatores, dentre os quais estão a doença de base, comorbidades pré-existentes e estágio de evolução da IRA. Neste estudo, observou-se que grande parte dos diagnósticos de base resultaram em causas de IRA prérenal de origem hipovolêmica, frente a isso, infere-se que a hidratação tenha sido a terapêutica de escolha, o que está de acordo com a literatura estudada (Prece et al, 2016; Cerqueira et al, 2014; Correa et al, 2020).

Após conduta médica especializada, verificou-se que 53,40\% receberam alta da UTI, 40\% evoluíram a óbito e 6,60\% necessitaram de transferência para UTI-Covid, devido coinfecção viral e necessidade de isolamento (Tabela 4). Observa-se que a taxa de evolução a óbito se assemelha aos demais estudos, como o de Peres et al. (2015), realizado com 152 pacientes, cuja mortalidade foi de $35 \%$. Ressalta-se, porém, que essa taxa varia entre 12 e $60 \%$ na literatura e isso se deve, primordialmente, a gravidade do paciente, as comorbidades e hábitos que possui, ao acometimento renal, bem como as múltiplas intervenções, a evolução clínica e prognóstico após terapêutica atribuída. 
Tabela 4: Classificação segundo KDIGO, tratamento e desfecho dos pacientes com IRA, Marabá, 2021.

\begin{tabular}{ccc}
\hline KDIGO & Fa & Fr \% \\
\hline Estágio 1 & 9 & $30 \%$ \\
Estágio 2 & 11 & $36,60 \%$ \\
Estágio 3 & 10 & $33,40 \%$ \\
\hline Tratamento & Fa & Fr \% \\
\hline Hidratação & 22 & $73,40 \%$ \\
Cirurgia & 1 & $3,30 \%$ \\
Diálise & 7 & $23,30 \%$ \\
\hline Desfecho & $\mathbf{F a}$ & $\mathbf{F r}$ \\
\hline Ala covid & 2 & $6,60 \%$ \\
Alta & 16 & $53,40 \%$ \\
Óbito & 12 & $40,00 \%$ \\
\hline
\end{tabular}

Fonte: Acervo da pesquisa.

Por fim, quando realizada uma análise entre o estágio que o indivíduo estudado fora classificado segundo o KDIGO, a presença de comorbidades e o desfecho deste (Tabela 5), verificou-se que daqueles classificados no Estagio 3 do KDIGO, 70\% possuíam hipertensão arterial sistêmica, $40 \%$ possuíam Diabetes mellitus e $60 \%$ foram a óbito, mostrando uma relação entre a gravidade da IRA, a presença de comorbidades e o desfecho negativo nesse estudo.

Isso corrobora com a literatura e também com os dados apresentados neste trabalho, uma vez que a injúria renal aguda é tão mais grave quanto maior o número de comorbidades, em especial a hipertensão arterial sistêmica e a diabetes mellitus (Kane-Gill et al, 2014; Cardoso et al, 2017; Lopes et al, 2018; Gaião et al, 2016; Chao-Yuan et al, 2020).

Logo, é fundamental compreender que pacientes críticos, internados em unidade de terapia intensiva devem ser conduzidos de forma rápida e assertiva afim de evitar um desfecho desfavorável (Luft et al, 2016; Lopes et al, 2018).

Tabela 5: Relação entre a gravidade da injuria renal aguda, presença de comorbidades e desfecho da amostra, Marabá, 2021.

\begin{tabular}{ccccccc}
\hline & KDIGO & HAS & DM & \multicolumn{3}{c}{ Desfecho } \\
\cline { 5 - 7 } & & & & Alta & Óbito & Ala Covid \\
\hline Estágio & Fa (Fr) & Fa (Fr) & Fa (Fr) & Fa (Fr) & Fa (Fr) & Fa (Fr) \\
\hline 1 & $9(30 \%)$ & $7(77,70 \%)$ & $5(55,50 \%)$ & $8(88,9 \%)$ & $0(0 \%)$ & $1(1,1 \%)$ \\
2 & $11(36,60 \%)$ & $7(63,60 \%)$ & $5(45,40 \%)$ & $7(63,70 \%)$ & $4(36,30 \%)$ & $0(0 \%)$ \\
3 & $10(33,40 \%)$ & $7(70 \%)$ & $4(40 \%)$ & $3(30 \%)$ & $6(60 \%)$ & $1(10 \%)$ \\
\hline
\end{tabular}

Fonte: Acervo da pesquisa.

\section{Considerações Finais}

Neste estudo, observou-se que o sexo prevalente foi masculino com idade acima de 55 anos e que o tempo de internação foi de até 7 dias. Constatou-se que indivíduos que tinham alguma doença crônica de base, em especial hipertensão arterial sistêmica e/ou diabetes mellitus e que tinham hábitos de vida não saudáveis, como o tabagismo obtiveram pior score na classificação KDIGO e apresentaram desfecho desfavorável.

Nesse sentido, compreender o perfil dos indivíduos atendidos no serviço pode favorecer melhor atendimento médico, com maior rapidez diagnóstica e permitir otimização terapêutica, afim de reduzir os impactos na morbimortalidade induzidos pela injúria renal aguda na unidade de terapia intensiva. 
Portanto, é fundamental que novos estudos sejam continuamente realizados, priorizando as particularidades clínicas dos indivíduos acometidos pela IRA em cada região do país, visto que o Brasil é uma nação multifacetada, com múltiplas culturas e hábitos que impactam diretamente no processo saúde-doença. Assim, será possível agregar a comunidade científica um maior contingente de subsídios técnicos capazes de diminuir os impactos gerados por esta patologia.

\section{Referências}

Bandiera, S. (2015). Alterações hepáticas e renais em ratos expostos ao álcool, fumaça de cigarro ou sua associação: Universidade Federal do Rio Grande do Sul.

Benichel, C. R. \& Meneguin, S. (2018). Fatores associados à lesão renal aguda em pacientes cirúrgicos na unidade de terapia intensiva. Revista da Rede de Enfermagem do Nordeste, 19 (1), 1-7

Cardoso, B. G., Carneiro, T. A. \& Magro, M. C. (2017). Recovery of patients with acute kidney injury requiring dialysis or not. Cogitare Enferm., 22(1), 1-9.

Cerqueira, D. P., Tavares, J. R. \& Machado, R. C. (2014). Fatores preditivos da insuficiência renal e algoritmo de controle e tratamento. Rev Latinoam Enferm., 22(2), 2117.

Chao-Yuan, H., Fabian, G. G., Marine, F. \& Geert, M. (2020). Modelos de predição clínica para lesão renal aguda na unidade de terapia intensiva: uma re visão sistemática. Rev. bras. ter. intensiva 32 (1), 1-9.

Corrêa, A. S. G., Coutinho, L. S., Jacoud, M. V. L., Carlos, A. R. \& Sória, D. A. C. (2020). Clinical manifestations and Nursing interventions in acute kidney injury in intensive care: an integrative review. Research, Society and Development, 9 (8), 12-20.

Dallacosta, F. M. \& Ferrari, T. (2018). Insuficiência renal aguda na uti: mortalidade e aspectos clínicos. XIII Semana Acadêmica e IV Mostra Científica de Enfermagem.

Duarte, F., Pessoa, E. A., Reis, L. A., Schor, N. \& Borges, F. T. (2016). Priming previne a insuficiência renal aguda nefrotóxica através da estimulação do mecanismo de defesa antioxidante. J Bras Nefrol., 38 (2), 161-172.

Estrela, C. (2018). Metodologia Científica: Ciência, Ensino, Pesquisa. Editora Artes Médicas.

Gaião, S. M., Gomes, A. A. \& Paiva, J. Á. (2016). Prognostics factors for mortality and renal recovery in critically ill patients with acute kidney injury and renal replacement therapy. Rev Bras Ter Intensiva. 28(1), 70-77.

Guedes, J. R., Silva, E. S., Carvalho, I. L. N. \& Oliveira, M. D. (2017). Incidência e fatores predisponentes de insuficiência renal aguda em unidade de terapia intensiva. Cogitare Enfermagem, 22 (2), 1-9.

Hsiao, J. \& Lima, A. F. C. (2015). Custo direto da hemodiálise em unidade de terapia intensiva adulto. Cogitare Enfermagem, 20 (4), 678-686.

Kane-Gill, S. L., Sileanu, F. E., Murugan, R., Trietley, G. S., Handler, S. M. \& Kellum, J. Á. (2014). Risk factors for acute kidney injury in older adults with critical illness: a retrospective e cohort study. Am J Kidney Dis., 65(6), 860-869.

Lopes, D., Scharan, L. D., Oliveira, J. L. C. O., Oliveira, R. B. S. R. \& Fernandes, L. M. (2018). Fatores de risco/causais para insuficiência renal aguda em adultos internados em terapia intensiva. Enf Bras., 17 (4), 1-10.

Luft, J., Boes, A. A., Lazzari, D. D., Nascimento, E. R. P., Busana, J. Á. \& Canever, B. P. (2016) Lesão renal aguda em unidade de terapia intensiva: características clínicas e desfecho. Cogitare Enfermagem, 21 (2), 1-9.

Ludke, M. \& Andre, M. E.D. A. (2013). Pesquisas em educação: uma abordagem qualitativa. São Paulo: E.P.U.

Orlandi, F. S., Lopes, J. M., Fukushima, R. L. M., Inouye, K. \& Pavarini, S. C. L. (2014) Qualidade de vida relacionada à saúde de pacientes renais crônicos em diálise. Acta Paul Enferm., 27 (3), 230-236.

Peres, L. A. B., Wandeur, V. \& Matsuo, T. (2015). Preditores de injúria renal aguda e de mortalidade em uma Unidade de Terapia Intensiva. J Bras Nefrol., 37(1), 38-46.

Prece, A., Cervantes, J., Mazur, C.S. \& Visentin, A. (2016). Perfil de pacientes em terapia intensiva: necessidade do conhecimento para organização do cuidado. Cad. da Esc. de Saúde, 2 (16), 35-48.

Rodriguez, A. H., Bub, M. B. C., Perão, O. F., Zandonadi, G. \& Rodriguez, M. J. H. (2016). Características epidemiológicas e causas de óbitos em pacientes internados em terapia intensiva. Rev. Bras. Enferm., 69 (2), 229-234.

Santos, E. S. \& Marinho, C. M. S. (2013). Principais causas de insuficiência renal aguda em unidades de terapia intensiva: intervenção de enfermagem. Rev. Enf., 3 (9), 1-8.

Sesso, R. C., Lopes, A. A., Thomé, F. S., Lugon, J. R. \& Martins, C. T. (2016). Inquérito Brasileiro de Diálise Crônica 2014. J Bras Nefrol., 38 (1), 54-61.

Souza, N. R., Freire, D. A., Souza, A. M. F. L., Lima, D. S., Brandão, C. S. \& Lima, V. S. B. (2017). Description of patientes with acute renal failure from na intensive therapy unit submitted to haemodialysis. Rev. Eletron. Cienc., 13 (2), 1-12.

Triquez, S. L. \& Dallacosta, F. M. (2012). Perfil dos pacientes com insuficiência renal aguda na unidade de terapia intensiva e principais diagnósticos de enfermagem. Unoesc \& Ciência-ACBS, 3(2), 123-130. 